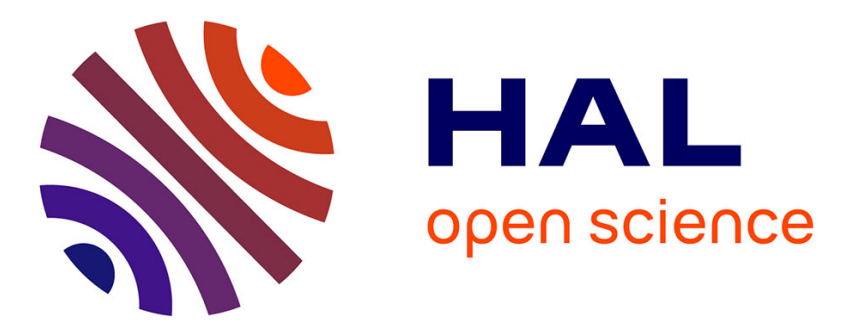

\title{
Anomalous fractional magnetic field diffusion through cross-section of a massive toroidal ferromagnetic core
}

\author{
Benjamin Ducharne, P. Tsafack, Y.A. Tene Deffo, B. Zhang, G. Sebald
}

\section{To cite this version:}

Benjamin Ducharne, P. Tsafack, Y.A. Tene Deffo, B. Zhang, G. Sebald. Anomalous fractional magnetic field diffusion through cross-section of a massive toroidal ferromagnetic core. Communications in Nonlinear Science and Numerical Simulation, 2021, 92, pp.105450. 10.1016/j.cnsns.2020.105450 . hal-03260447

\section{HAL Id: hal-03260447 https://hal.science/hal-03260447}

Submitted on 15 Jun 2021

HAL is a multi-disciplinary open access archive for the deposit and dissemination of scientific research documents, whether they are published or not. The documents may come from teaching and research institutions in France or abroad, or from public or private research centers.
L'archive ouverte pluridisciplinaire HAL, est destinée au dépôt et à la diffusion de documents scientifiques de niveau recherche, publiés ou non, émanant des établissements d'enseignement et de recherche français ou étrangers, des laboratoires publics ou privés. 


\title{
Anomalous fractional magnetic field diffusion through cross-section of a massive toroidal ferromagnetic core
}

\author{
B. Ducharne ${ }^{a}$, P. Tsafack ${ }^{b}$, Y.A. Tene Deffoc, B. Zhang ${ }^{d}$, G. Sebald ${ }^{\text {e }}$ \\ a Univ. Lyon, INSA-Lyon, LGEF EA682, F69621 Villeurbanne, France \\ ${ }^{b}$ Faculty of Engineering and Technology, University of Buea, Buea, Cameroon \\ ${ }^{c}$ Green Manufacturing R\&D Laboratory, School of Mechanical, Electrical and Information \\ Engineering, Shandong University, Weihai, China \\ d ELyTMaX UMI 3757, CNRS - Université de Lyon - Tohoku University, International Joint Unit, \\ Tohoku University, Sendai, Japan
}




\section{Abstract}

Toroidal massive ferromagnetic cores are used in a wide range of electromagnetic applications, such as current sensors, inductances, static converters and filters. Growing interest exists in the industrial field with regards simulation tools to reduce experimental campaigns and improve product knowledge and performance. Accurate simulation results require a consideration of precise electromagnetic laws, such as the exact non-linear magnetic behavior of toroidal magnetic cores. Under the influence of an external surface magnetic field that was created by a surrounding coil, the local magnetic state through a ferromagnetic core cross-section was ruled by a combination of magnetic domain kinetics and external magnetic field diffusion. Conventional methods to simulate magnetic behavior are based on a separation of magnetic contributions, where microscopic Eddy currents from domain wall motions and macroscopic currents from external magnetic field variations are considered separately. This separation is artificial, because both loss mechanisms occur simultaneously and interact. In this study, an alternative solution was proposed through the resolution of a two-dimensional anomalous fractional magnetic field diffusion. The fractional order constitutes an additional degree of freedom in the simulation scheme, which can be identified by comparison with the experimental results. By adjusting this order, accurate local and global simulation results can be obtained on a broad frequency bandwidth and allow for the precise prediction of the dynamic magnetic behavior of a toroidal massive magnetic core.

\section{Keywords}

Anomalous magnetic diffusion, fractional derivative, toroidal magnetic core, magnetic hysteresis. 


\section{1 - Introduction}

The working principles of electromagnetic devices rely on magnetic/electric and electric/magnetic energy conversions [1]. A large amount of energy is converted in applications, including power transformers, quasi-static converters and electric motors. Small amounts are involved in applications, such as in communication support, i.e., sensor-type applications [2]. To improve the conversion efficiency and/or accuracy of the electromagnetic devices, the magnetic field needs a magnetic support to be guided in spatial areas where multiphysics conversions occur. This magnetic support is always made from a soft ferromagnetic circuit that is characterized by a high magnetic permeability, high saturation levels and weak coercive fields. Toroidal massive magnetic cores are popular in applications where the desirable features are a high specific power per mass and volume and minimal electromagnetic interferences [3]. The main drawback that limits their use for general purpose applications is the inherent difficulty of winding wire through the center of a torus. The high non-linear magnetic behavior of the ferromagnetic core is another issue that needs to be addressed. Under the influence of an external magnetic field, the magnetic state of a toroidal core exhibits hysteresis, saturation, frequency dependence and an inhomogeneous distribution. A precise knowledge of these magnetic properties and behaviors is the only way to reach electromagnetic devices of upgraded performances [4].

In typical toroidal core applications, the coil is wound through a hole in the torus and around its outside

(Fig. 1). The ideal coil was distributed evenly around the circumference of the torus. The symmetry of the coil geometry and the electrical current that was passing through the coil produced a surface tangential magnetic field of a circular shape on the periphery of the magnetic core. The lack of sharp bends helps to lock the magnetic induction field inside the ring specimen. The toroidal geometry improves the conversion ratio and reduces the electromagnetic interference that is radiated by the coil [5]. 


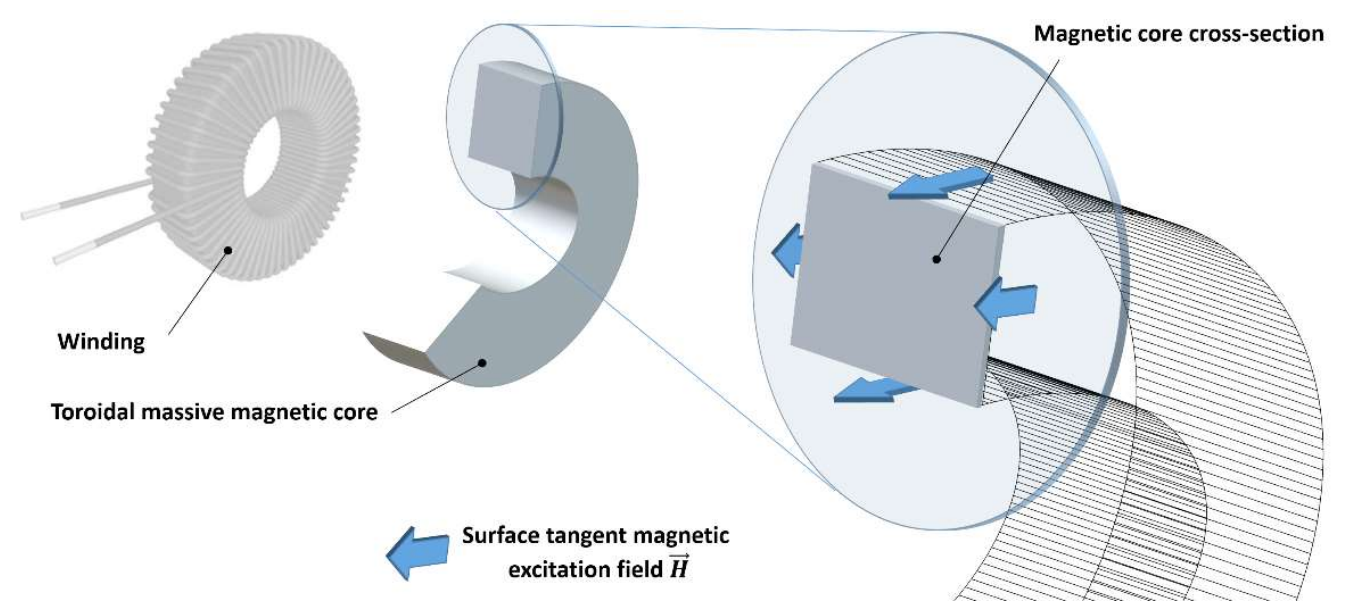

Fig. 1 - Three-dimensional illustration of a toroidal magnetic circuit, including the winding and the magnetic core.

For decades, industry and academic researchers have collaborated to propose simulation tools to reproduce the magnetic behavior of massive toroidal ferromagnetic cores precisely [6]. This problem is complex because magnetism originates at the atomic level and the macroscopic magnetic behaviors are influenced strongly by interactions inside the mater and at different space scales [7][8]. Standard approaches for magnetic behavior simulation are based on the so-called separation losses principle [9][10]. This principle consists of separating the magnetic state of a ferromagnetic component under the influence of a dynamic magnetic excitation into three loss terms:

\section{_ Hysteresis losses:}

This magnetic contribution results from the magnetic domain kinetics, expansions and contractions that occur during magnetization. These movements lead to losses as the domain walls get pinned on defects in the crystal structures and unpinned under the influence of stronger magnetic excitation levels, and dissipate energy as heat. Because the energy that is lost in each magnetization cycle is constant, the power of this contribution is proportional to the frequency.

_ Eddy-current losses:

If the toroidal ferromagnetic core is electrically conductive, the changing magnetic field induces circulating loops of current in the core, which is termed macroscopic Eddy currents (as opposed to the microscopic currents that are linked to the domain wall motions). These macroscopic Eddy currents result from electromagnetic induction. The loops are perpendicular to the magnetic field axis. The 
current energy is dissipated as heat in the resistance of the core material. The power loss is proportional to the area of the loops and inversely proportional to the resistivity of the core material. The power of this contribution is proportional to the square of the frequency.

_ Anomalous losses:

This contribution includes any losses that are not included in either the eddy-current or the hysteresis losses. Anomalous losses can also be observed through a broadening of the hysteresis loop with frequency. Physical mechanisms for anomalous loss include localized eddy-current effects near moving domain walls.

The separation principle is well-known in the scientific community, because it is simple and accurate enough in a wide range of applications. The separation principle also exhibits strong limitations, including its restriction to harmonic excitation, which makes it particularly inappropriate for application to time-dependent simulations, because it only provides average information. The general idea of separating the hysteresis losses contradicts the physical nature of the magnetization processes where different phenomena interact simultaneously at different scales.

Though rarely mentioned, models that combine all contributions exist in the literature. In [11], a simultaneous resolution scheme is proposed for ferromagnetic lamination, where a one-dimensional (1D) modified diffusion magnetic field equation is solved. The time derivative term was replaced by a dynamic material law that lead to a strong formulation that can be solved through finite differences. This simulation approach allows access to local information. A similar approach has been proposed in [12] for the two-dimensional (2D) simulation of the magnetic state through the cross-section of a toroidal massive magnetic core. In this article, the ring specimen was under the influence of the magnetic field that was created by a winding even distribution. The magnetic skin effect was highlighted. This combined resolution has multiple advantages:

_ it can be solved in the time domain and the local information can be used to design the magnetic core with a reasonably good accuracy. 
_ It can also be used as a pedagogic tool to explain the dynamic magnetization processes in a ferromagnetic core.

_ However, it also exhibits some significant drawbacks, including the limitation to given geometries where sparse finite-difference discretization is sufficient to obtain correct simulation behaviors. A combination of the implicit nature of the material law and the strong nonlinearity of hysteresis often leads to convergence issues, which forces a reduction in simulation step time and, in many cases, results in an abortion of the simulation.

In [8], an alternative elegant solution has been proposed to retain the benefits and address the convergences issues. The dynamic effect during the magnetization cycles could be interpreted in term of an anomalous diffusion process. The Weiss domain distribution and movements distort the magnetic diffusion process, which becomes anomalous. Also in [8], the anomalous diffusion equation is described by replacing the first-order time derivation by a fractional-order derivative in the magnetic diffusion equation (Eq. 1):

$$
\nabla^{2} \vec{H}=\sigma \cdot \frac{d^{\alpha} \vec{B}}{d t^{\alpha}} \Rightarrow \frac{\partial^{2} \overrightarrow{H(x, t)}}{\partial x^{2}}=\sigma \cdot \frac{d^{\alpha} \overrightarrow{B(x, t)}}{d t^{\alpha}}
$$

Fractional calculus has been associated with electromagnetism [13]. In [14], the Maxwell laws were extended to non-integer-order differential calculus. When applied to transmission lines, electrical motors and transformers allow us to consider the skin effect precisely. In [15], fractional integral and differential operators are used to describe the frequency-dependent Havriliak-Negami dielectric relaxation models in the time-domain. In [16] and [17], an additional fractional contribution is used to consider the dynamic polarization effect in a piezo-ceramic.

The simulation method described in [8] is promising. However, this study is restricted to magnetic laminations where correct simulation results were obtained by limiting the resolution to one dimension only.

In this article, a similar method was proposed and extended to a 2D resolution to simulate the dynamic magnetic state through a toroidal magnetic core cross-section. 


$$
\nabla^{2} \vec{H}=\sigma \cdot \frac{d^{\alpha} \vec{B}}{d t^{\alpha}} \Rightarrow \frac{\partial^{2} \overrightarrow{H(x, y, t)}}{\partial x^{2}}+\frac{\partial^{2} \overrightarrow{H(x, y, t)}}{\partial y^{2}}=\sigma \cdot \frac{d^{\alpha} \overrightarrow{B(x, y, t)}}{d t^{\alpha}}
$$

The simulation method is defined in Section 2 before the experimental setup and tested samples are described. Comparative simulations and measures are provided and used to validate our theory. Additional simulation results are provided in the conclusions, which provides information on the simulation parameters ( $\sigma$ is the electrical conductivity and $\alpha$ is the fractional derivative order), and their influences.

\section{2 - Simulation method}

\section{a) Numerical resolution of $2 \mathrm{D}$ anomalous magnetic field diffusion equation}

Similar to [11][12][18], finite differences were chosen for the spatial resolution of an anomalous diffusion equation.

$$
\left\{\begin{array}{l}
\nabla^{2} \vec{H}=\frac{\partial^{2} \overline{H(x, y, t)}}{\partial x^{2}}+\frac{\partial^{2} \overline{H(x, y, t)}}{\partial y^{2}}=\sigma \cdot \frac{d^{\alpha} \overline{B(x, y, t)}}{d t^{\alpha}} \\
\text { at nodei: } \\
\frac{-H\left(x_{i-1}, y_{i}, t\right)+2 \cdot H\left(x_{i}, y_{i}, t\right)-H\left(x_{i+1}, y_{i}, t\right)}{e^{2}}+\frac{-H\left(x_{i}, y_{i-1}, t\right)+2 \cdot H\left(x_{i}, y_{i}, t\right)-H\left(x_{i}, y_{i+1}, t\right)}{e^{2}}=\sigma \cdot \frac{d^{\alpha} B\left(x_{i}, y_{i}, t\right)}{d t^{\alpha}}
\end{array}\right.
$$

(3)

For symmetry reasons, half of the magnetic core cross section was meshed (see Fig. 2). 


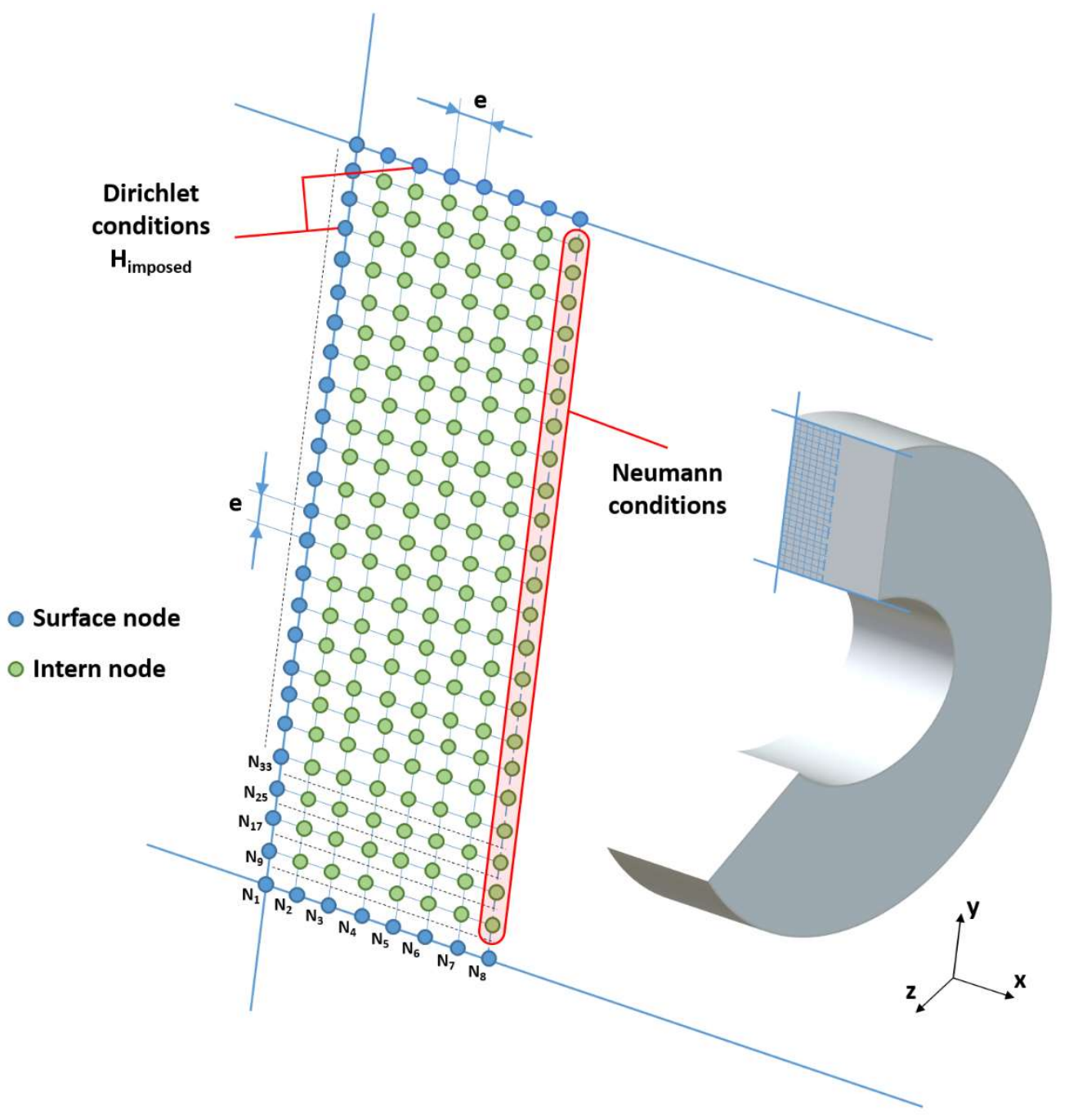

Fig. 2 - Finite-difference space discretization, boundary conditions.

The surface-excitation field $\mathrm{H}$ was imposed on the blue nodes (Dirichlet boundary conditions, Fig. 2). Under electrical-current-imposed conditions, $\mathrm{H}_{\mathrm{i}}$, which is the excitation field for each blue node $\mathrm{i}$ was derived from the Ampere theorem:

$$
H_{i}(t)=\frac{N \cdot I(t)}{2 \pi \cdot r_{i}}
$$

where $\mathrm{N}$ is the number of turns, $\mathrm{l}(\mathrm{t})$ is the time-dependent electrical current and $\mathrm{r}_{\mathrm{i}}$ is the radius distance between nodes $i$ and the magnetic core rotation axis. Neumann boundary conditions were set on the red zone nodes (Fig. 2) to consider the symmetry property.

\section{b) Fractional derivative of Induction field B}


Anomalous diffusion processes in complex media can be considered by using fractional-order diffusion equation models. A fractional derivative operator as the time-dependent term (as is the case in Eq. 1) corresponds to a long heavy tail decay. Fractional calculus is a branch of mathematical analysis that is dedicated to the study of non-integer-order derivatives. The order of the derivative can be a real or a complex number. The $\alpha$ derivative of a function $f(t)$ at a given time $\mathrm{t}$ is a local property only when $\alpha$ is an integer; which is not the case for non-integer power derivatives. It is incorrect to say that the fractional derivative at $t$ of a function $f(t)$ depends only on the values of $f$ near $t$, in the way that integerpower derivatives do. A clear parallel can be established between the influence of the time history in the resolution of a time-fractional derivative and the influence of the time history in the hysteretic property of a ferromagnetic material. This observation can be used for the physical interpretation of fractional derivatives.

Many definitions have been proposed to describe fractional derivatives, such as those by Liouville [19][21] (Eq. 5), Riemann-Liouville [22], Grünwald-Letnikov [23][24] and Caputo [25].

$$
\frac{d^{\alpha} f(t)}{d t^{\alpha}}=D^{\alpha}[f(t)]=\frac{1}{\Gamma(1-\alpha)} \frac{d}{d t} \int_{-\infty}^{t}(t-\tau)^{-\alpha} f(\tau) d \tau, \quad-\infty<t<+\infty
$$

Equation 5 can be interpreted as the convolution between $f(\mathrm{t})$ and $\mathrm{t}^{\alpha} \mathrm{H}(\mathrm{t}) / \Gamma(1-\alpha)$ where $\Gamma(\alpha)$ is the gamma function. In the Fourier domain, where every signal is harmonic and where a first-order derivative is a jw multiplication, fractional derivatives become a $(j \omega)^{\alpha}$ multiplication [26]-[28]. For the numerical resolution of our anomalous diffusion equation, two options have been tested:

_ The direct Grünwald-Letnikov derivative definition:

$$
\begin{aligned}
& \frac{d^{\alpha} f(t)}{d t^{\alpha}}=D^{\alpha} f(t)=\lim _{h \rightarrow 0} \frac{1}{h^{\alpha}} \sum_{k=0}^{\infty}(-1)^{k}\left(\begin{array}{l}
\alpha \\
k
\end{array}\right) f(t-k h) \\
& \left(\begin{array}{l}
\alpha \\
k
\end{array}\right)=\frac{\Gamma(\alpha+1)}{\Gamma(k+1) \Gamma(\alpha+1-k)}
\end{aligned}
$$

_ A convolution approach:

$$
\frac{d^{\alpha} f(t)}{d t^{\alpha}}=\frac{d^{m} f(t)}{d t^{m}} * \frac{1}{\Gamma(m-\alpha)} \frac{1}{t^{\alpha+1-m}}
$$

(Convolution of derivative of order, first integer $m$ larger than $\alpha$ and a memory function). 
The Matlab ${ }^{\circledR}$ convolution function has been used for this resolution, but better results were obtained with the Grünwald-Letnikov definition.

\section{c) Combined resolution}

A combination of Eqs. 3 and 6 allows the equation to be solved for each node of the space discretization window:

$\frac{-H\left(x_{i-1}, y_{i}, t\right)+2 \cdot H\left(x_{i}, y_{i}, t\right)-H\left(x_{i+1}, y_{i}, t\right)}{e^{2}}+\frac{-H\left(x_{i}, y_{i-1}, t\right)+2 \cdot H\left(x_{i}, y_{i}, t\right)-H\left(x_{i}, y_{i+1}, t\right)}{e^{2}}=\sigma \cdot \lim _{h \rightarrow 0} \frac{1}{h^{\alpha}} \sum_{k=0}^{\infty}(-1)^{k}\left(\begin{array}{c}\alpha \\ k\end{array}\right) B\left(x_{i}, y_{i}, t-k h\right)$

A matrix system can be established by using every equation (Eq. 9).

$$
[M] \cdot[X]=[S]
$$

$M$ is the stiffness matrix, $X$ is unknown vector $H_{i}$ and $S$ is a vector that is filled with the remaining terms.

The matrix system construction is like the $1 \mathrm{D}$ system in [8]. Boundary nodes where Dirichlet or Neumann-type conditions are imposed according to their geometrical position must be considered (see Fig. 2). $\mathrm{B}_{\mathrm{i}}$ and $\mathrm{H}_{\mathrm{i}}$ are connected locally through an anhysteretic relationship that is described by a hyperbolic sigmoid function:

$$
B_{i}^{a n h}=B_{s} \tan ^{-1}\left(\frac{H_{i}}{a}\right)
$$

$B_{i}$ is the local induction, $B_{s}$ the saturation induction and $a$ is an anhysteretic induction trajectory parameter. Equation 3 is not used "as if" in the simulation but is used through the related permeability, which is expressed as a function of $B_{i}$ :

$$
\mu_{i}^{a n h}\left(B_{i}^{a n h}\right)=\frac{B_{s} \cdot a}{\left(1+\tan ^{2}\left(\frac{B_{i}^{a n h}}{B_{s}}\right)\right)}
$$

The implicit nature of Eq. 8 is an issue. When combined with the non-linear behavior of Eq. 3, Eq. 8 sometimes leads to divergences under a high magnetic excitation field, whereas the magnetic core is saturated. In the final version of the code, the issue is solved through two methods: 
- In the first term of the $B_{i}$ series in Eq. 8, the permeability $\mu_{i}$ is evaluated based on the previously calculated $B_{i}(t-d t)$.

- We force the local anhysteretic permeability to vary monotonically with the magnetic excitation. For every simulation step, a test is executed if the monotonic property is not respected, and we impose $B_{i}^{a n h}(t)=B_{i}^{\text {anh }}(t-d t)$.

Figure 3 depicts the evolution of average dynamic hysteresis losses through the cross section of a toroidal magnetic core as a function of $f, \alpha$, and for different values of electrical conductivity. Matrix system (9) is solved and harmonic-type current excitation is imposed. The average induction is calculated from Eq. 12:

$$
B=\frac{\sum_{1}^{n} B_{i}}{n}
$$

where $n$ is the number of nodes. The dynamic hysteresis losses $<A>$ are calculated through an integration of the resulting hysteresis loop (Eq. 13):

$$
<A>=\int_{0}^{T} B(t) \cdot d H(t)
$$

As observed in Fig. 3, the dynamic losses that were simulated with the anomalous fractional diffusion equation were negligible in the frequency range $f$ and the fractional order $\alpha$ tested. If the electrical conductivity $\sigma$ was weak, the emergence of Eddy currents were weak too. For higher levels of $\sigma$ and under an increasing sweep of frequency $f$, the dynamic losses show a Gaussian-like shape, which shows that an average value and standard deviation increase as $\alpha$ decreases. Similar results have already been observed and plotted in Fig. 4 in [8] for magnetic laminations where a 1D equation was solved. 

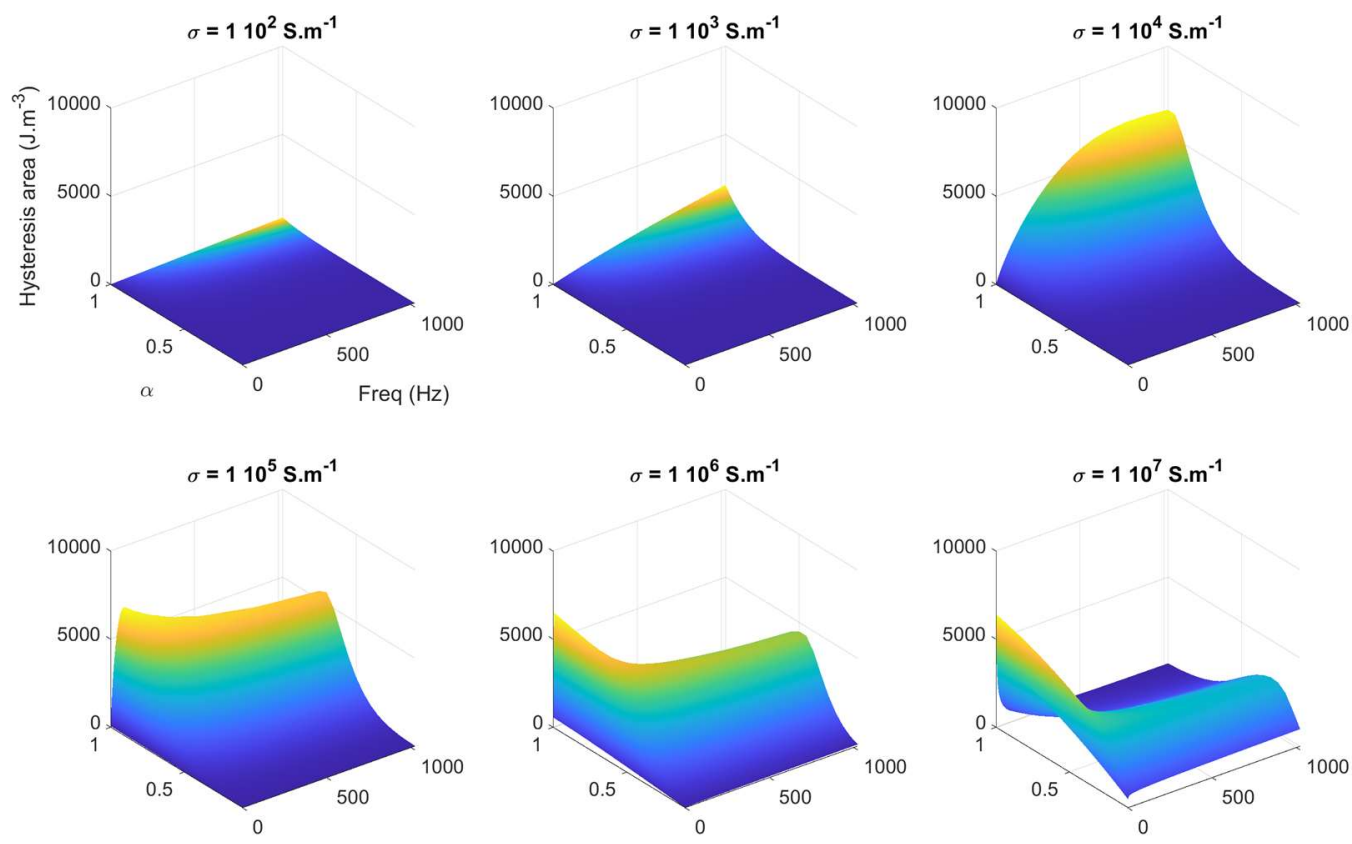

Fig. 3 - Hysteresis area as a function of $\alpha$ and $f$ for an electrical conductivity from $110^{2}$ to $110^{7} \mathrm{~S} . \mathrm{m}^{-1}$.

Figure 4 depicts the magnetic field distribution through the cross-section of the toroidal massive ferromagnetic core for different values of $f, \alpha$ and $\sigma$. A full magnetization cycle was simulated and the color scale was set to a maximum of the resulting local induction fields $\mathrm{B}_{\mathrm{i}}$. As expected, for a weak $\sigma$, the magnetic field distribution was homogeneous. At a higher conductivity level, $\alpha$ was close to one with large frequency values, and the skin effect (rejection of Eddy currents over the cross-sectional peripheral areas) was obvious. The Eddy currents acted as a shield and restricted the magnetic field in a peripheral belt of magnetic material with a width that decreased as the frequency increased. The corresponding average hysteresis cycles $\mathrm{B}\left(\mathrm{H}_{\text {surf }}\right)$ are plotted as well, where $\mathrm{H}_{\text {surf }}$ is the tangent magnetic excitation field that is imposed on the superior surface of the magnetic core. If $\sigma$ and $\alpha$ are weak, the hysteresis area is limited. The hysteresis area is limited too for high values of $\sigma$ and $f$ where the limitation of the magnetic field penetration prevents magnetization, and the resulting hysteresis loop shows reduced $B$ values. 

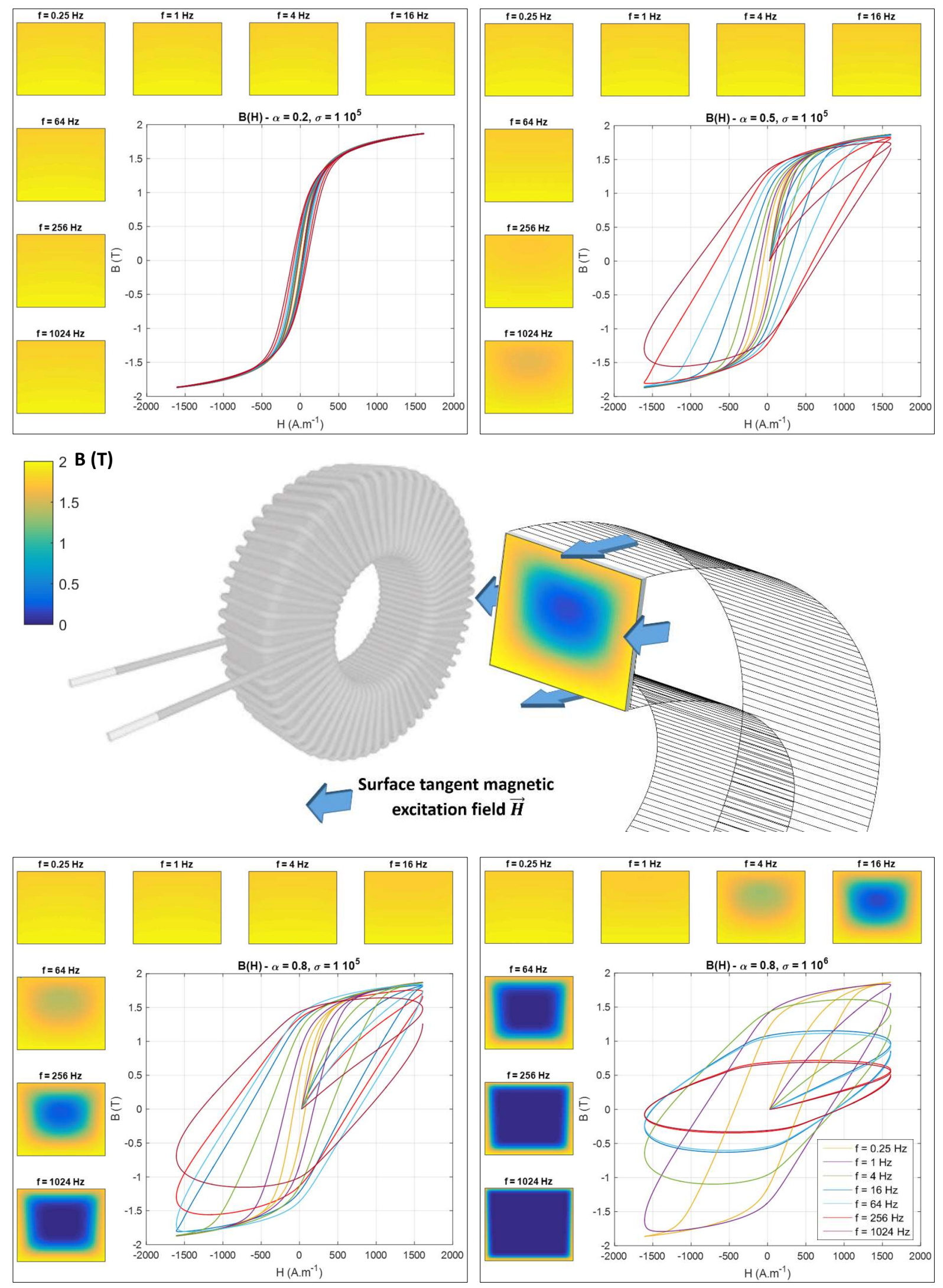

Fig. 4 - Magnetic state distribution and hysteresis cycles for different values of $\alpha, \sigma$ and $f$. 
Figures 5 and 6 illustrate the effect of $\alpha$, which is the fractional calculus order. The frequency $f$ and the electrical conductivity $\sigma$ were set to $64 \mathrm{~Hz}$ and $110^{5} \mathrm{~S} \cdot \mathrm{m}^{-1}$, respectively. $\alpha$ increases by 0.2 . The first $\alpha$ that was tested was 0.2 and the last simulation corresponded to the integer-order modelling.
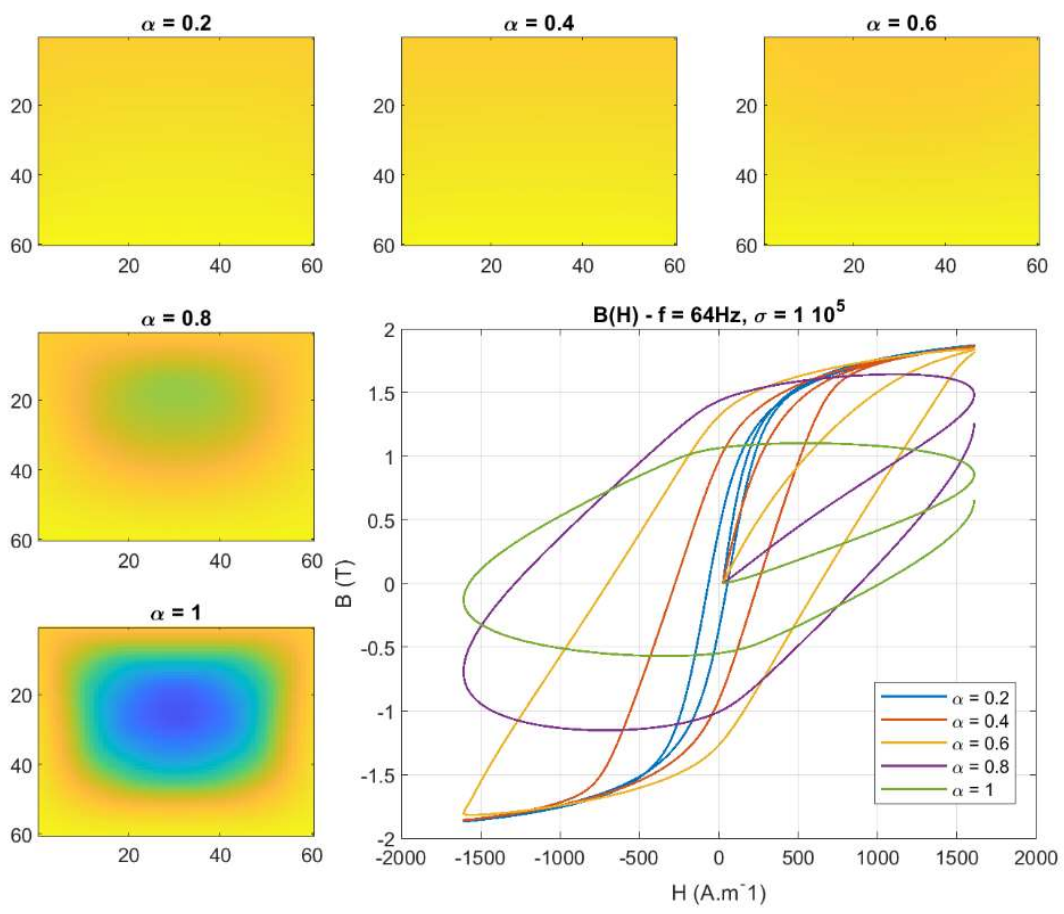

Fig. 5 - Magnetic state distribution and hysteresis cycles for different values of $\alpha$ ( $\sigma=110^{5}$ and $f=64 \mathrm{~Hz}$ ).

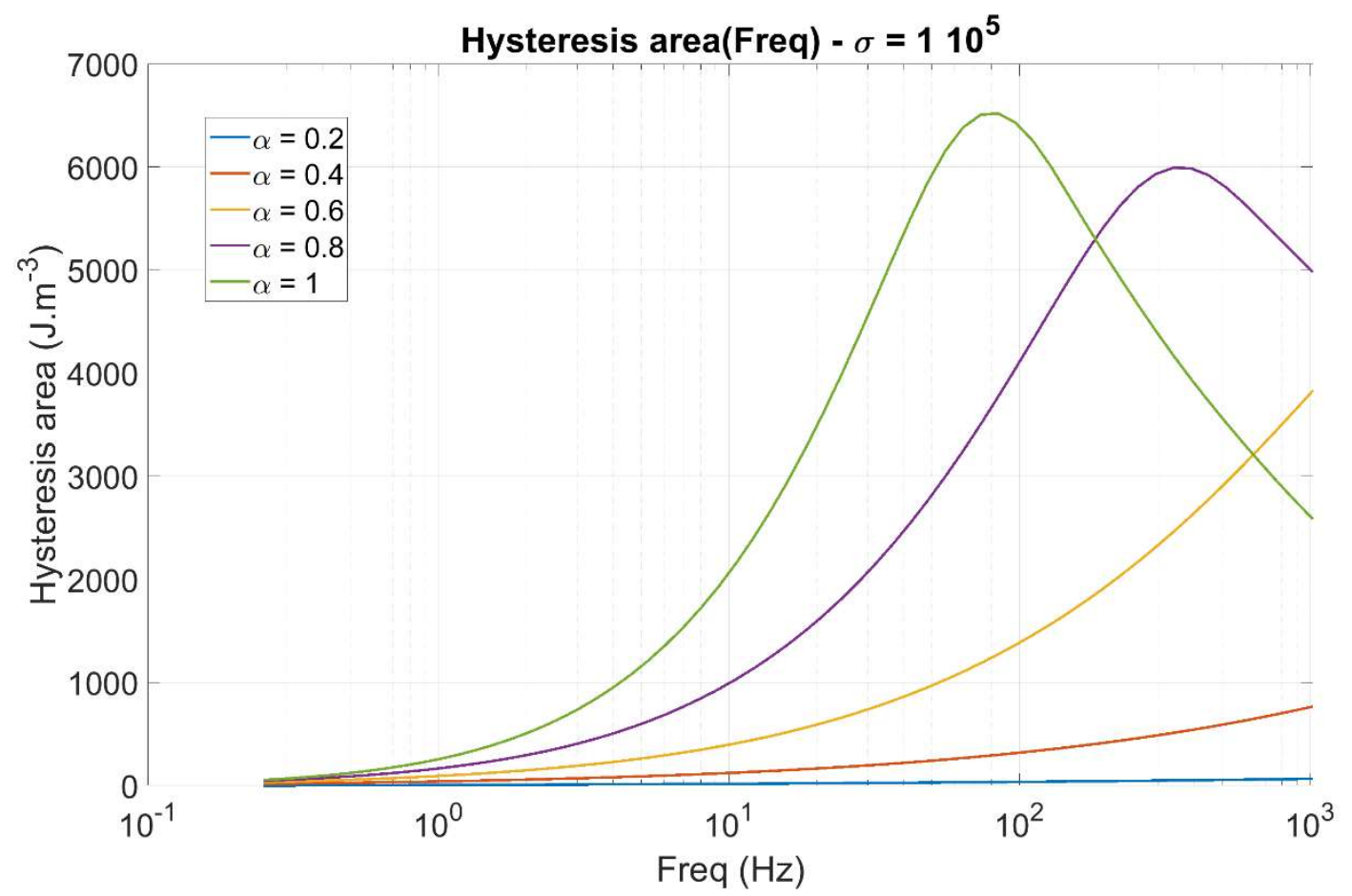

Fig. 6 - Hysteresis area as a function of $f$ for different values of $\alpha\left(\sigma=110^{5}\right.$ and $f=64 \mathrm{~Hz}$ ). 
For a given frequency and conductivity, a small $\alpha$ reduces the diffusion process, which limits the development of macroscopic Eddy currents and the hysteresis loop shape remains close to the anhysteretic behavior. By increasing $\alpha$, we activate the diffusion process and its consequences (i.e., the spread of the macroscopic Eddy currents and large variations of the hysteresis area). The electrical conductivity cannot be considered as a simulation degree of freedom because it is a physical property that is intrinsic to the tested specimen. The frequency $f$ is imposed by the magnetic field excitation. An integer order leads to incorrect simulation results because it does not consider the influence of the magnetic domain wall motions and their related microscopic Eddy currents. It appears impossible to quantify this influence precisely, however, we know that they modify the diffusion process and results in anomalous behaviors. An integer-order diffusion process gives correct simulation results when the media is homogeneous. Because of the presence and organization of the magnetic domains, a ferromagnetic massive toroidal specimen is magnetically strongly inhomogeneous. The only way to simulate the magnetization process correctly is to balance the diffusion process differently compared with the integer-order classic process. By replacing the first-order derivative with a fractional order provides flexibility in the simulation of the diffusion process. By adjusting $\alpha$, excellent matches could be obtained with the experimental results.

\section{4 - Experimental validation}

\section{a) Description of experimental setup}

A dedicated experimental setup was used to validate the simulation results. A three-dimensional (3D) overview of the setup is shown in Fig. 7: 


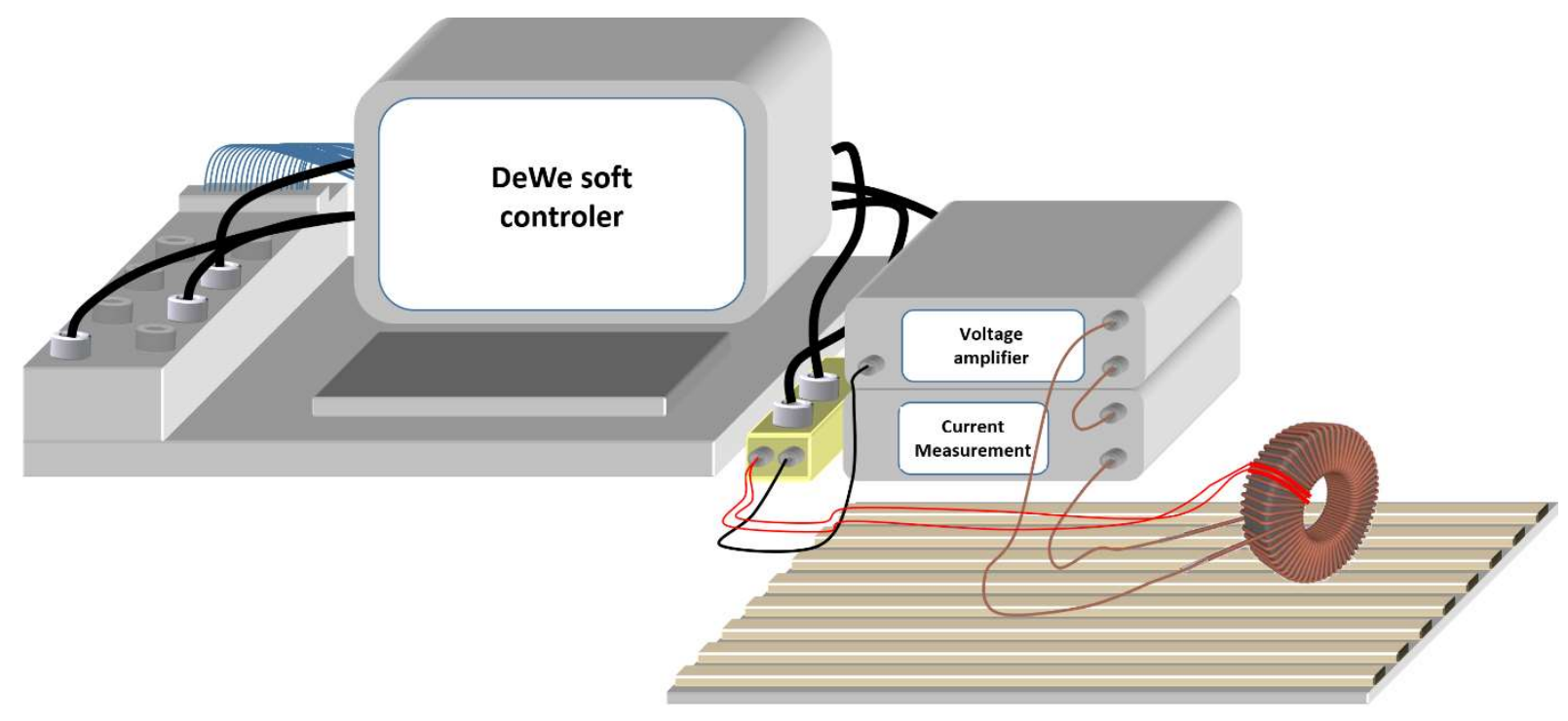

Fig. 7-3D overview of experimental setup.

Measurements were performed according to standard IEC 60604-6 [29]. A 200-turns-surrounding coil constituted the primary excitation. A local 10-turns-surrounding sensor coil was used to measure the magnetic flux locally through the cross section of the magnetic core. Harmonic-type-B imposed characterization was achieved as recommended in [29]. Real-time feedback control was ensured from DEWESoftX2 data acquisition software that was combined with a SIRIUSif $8 \times$ CAN data-acquisition/generation card. These devices ensured that feedback control of the magnetizing current was provided simultaneously to acquire measured signals. The surface tangent excitation field $\mathrm{H}$ was calculated from Eq. 4 and from the primary coil current measurement. A numerical integration of the surrounding coil sensor signal was used to plot the induction field variations. A numerical correction was performed to remove the undesired drift because of the integration step. Before each experimental test, the reproducibility of the results was ensured by a demagnetization of the tested specimens. The excitation coil was subjected to a slowly decreasing amplitude and a $50-\mathrm{Hz}$ sinusoidal electrical current. This process ensured a full demagnetization under a controlled magnetic excitation rate. A full demagnetization procedure lasted slightly less than $1 \mathrm{~min}$.

\section{b) Description of tested samples}


The tested samples were a 40NCD7 ferromagnetic structural steel that is used extensively in shipping, vehicle, airplane, railway, bridge, pressure vessel, machine tool and mechanical component fields with a large cross-sectional size. The chemical composition of the 40NCD7 is provided in Table 1.

Table 1 - Chemical composition, mass fraction in \% of 40NCD7.

\begin{tabular}{c|c|c|c|c|c|c|c}
$\mathbf{C}(\%)$ & $\mathbf{M n}(\%)$ & Si (\%) & $\mathbf{P}(\%)$ & $\mathbf{S}(\%)$ & $\mathbf{C r}(\%)$ & $\mathbf{N i}(\%)$ & Mo (\%) \\
\hline $\min : 0.38$ & $\min : 0.65$ & $\min : 0.15$ & 0.025 & 0.025 & $\min : 0.7$ & $\min : 1.65$ & $\min : 0.2$ \\
$\max : 0.43$ & $\max 0.85$ & $\max : 0.35$ & & & $\max 0.9$ & $\max : 2$ & $\max : 0.3$
\end{tabular}

The electrical conductivity is relatively high, $410^{6} \mathrm{~S} \cdot \mathrm{m}^{-1}<\sigma<510^{6} \mathrm{~S} \cdot \mathrm{m}^{-1}$. Combined with the massive section of the toroidal core, dynamic effects can be observed even at low frequency levels. The test specimen dimensions are shown in Fig. 8. The specimen thickness was $10 \mathrm{~mm}$.
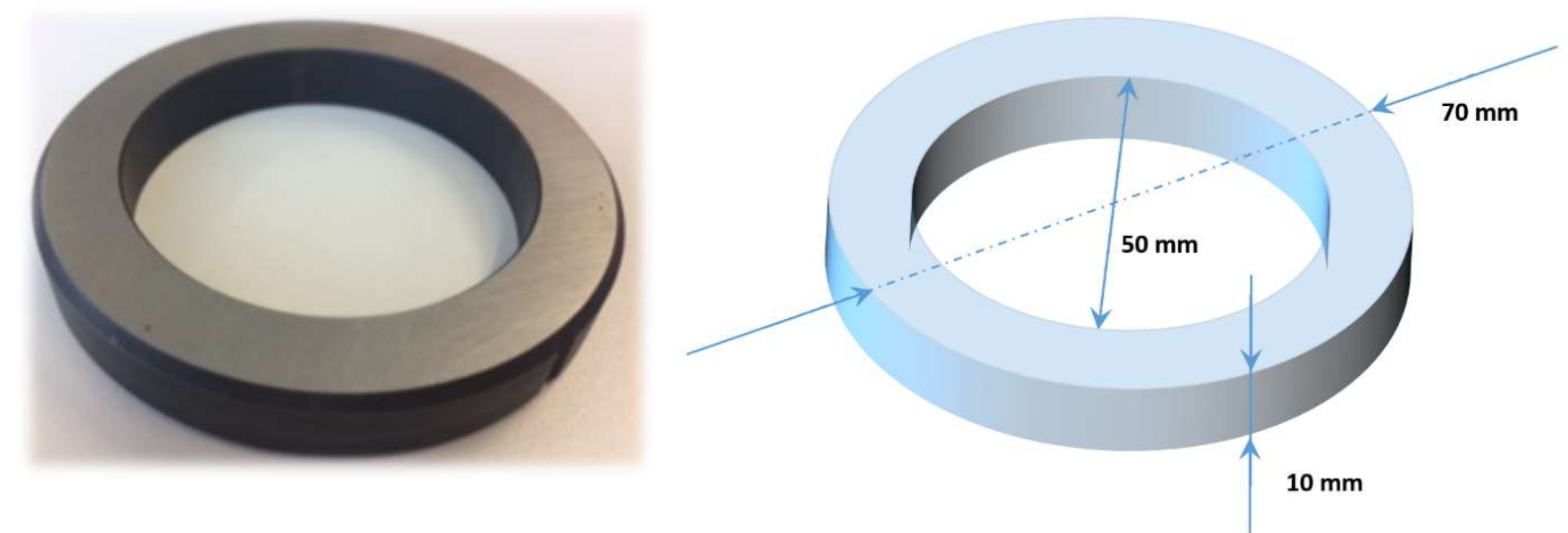

Fig. 8 - Tested specimen dimensions.

A tempering stage was used to reduce the residual stresses because of the machining operations. For this study, we selected $40 \mathrm{NCD} 7$ over $100 \mathrm{Cr} 6$ and M500NIL. The 40 NCD7 exhibited the most homogeneous mechanical, electrical and magnetic properties.

\section{c) Comparative simulations/measures}

According to the IEC 60604-6 standard on ring specimens, a correct characterization of the magnetic signature needs to be performed under a "B-imposed" sinus-type waveform [24]. The fractional diffusion equation resolution as described in this study implies knowledge of the surface magnetic excitation field $\mathrm{H}$ that surrounds the magnetic ring. To address this issue and to converge to the correct 
simulation results even under B imposed conditions, a feedback simulation tool that includes a proportional/integral corrector was used. This solution has already been used for magnetic lamination in [8]. The corresponding bloc scheme is depicted in Fig. 9.

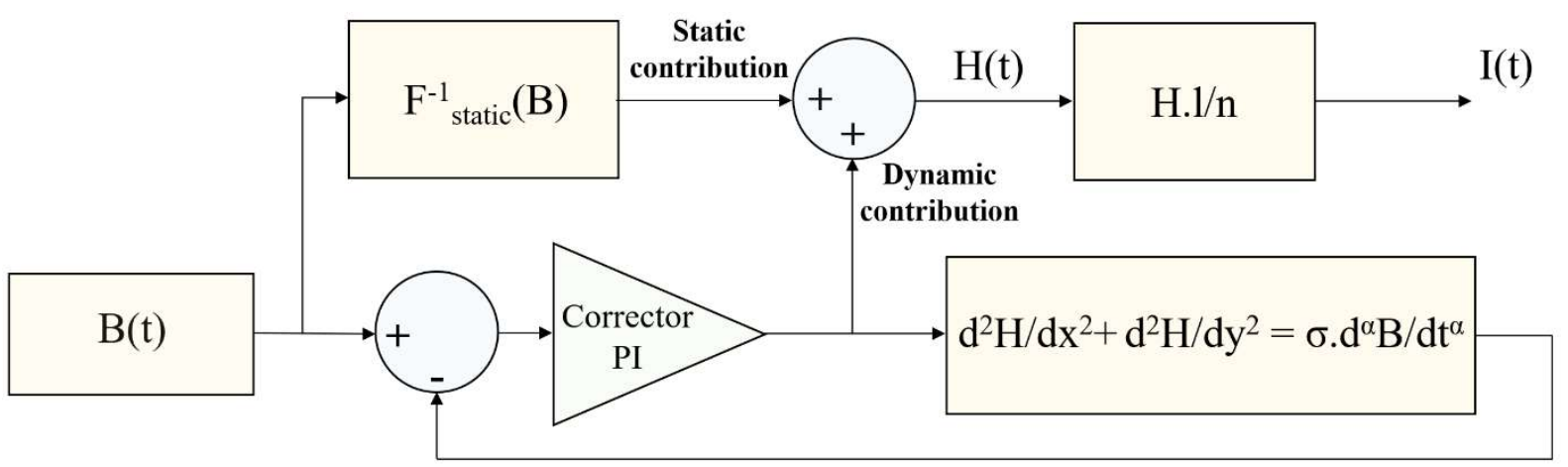

Fig. 9 - Simulation bloc scheme that includes the quasi-static and dynamic contribution.

The corrector parameters were set from the numerical optimization. To compare the simulation and experimental results, a static contribution was added to the fractional diffusion equation. This contribution corresponds to the hysteretic behavior that can be observed in a quasi-static state, i.e., below the quasi-static frequency threshold. Working under "B-imposed" conditions simplifies the quasi-static contribution in our simulation. As in [27][28] or [30], the classic Preisach and Jiles-Atherton (J-A) models in their inverse versions [31]-[33] can be used. Figure 10 compares the simulations/measurements for the 40 NCD7. The simulation results were obtained by using the simulation scheme in Fig. 9 and a 1.6 T average imposed sinus induction state was imposed. 

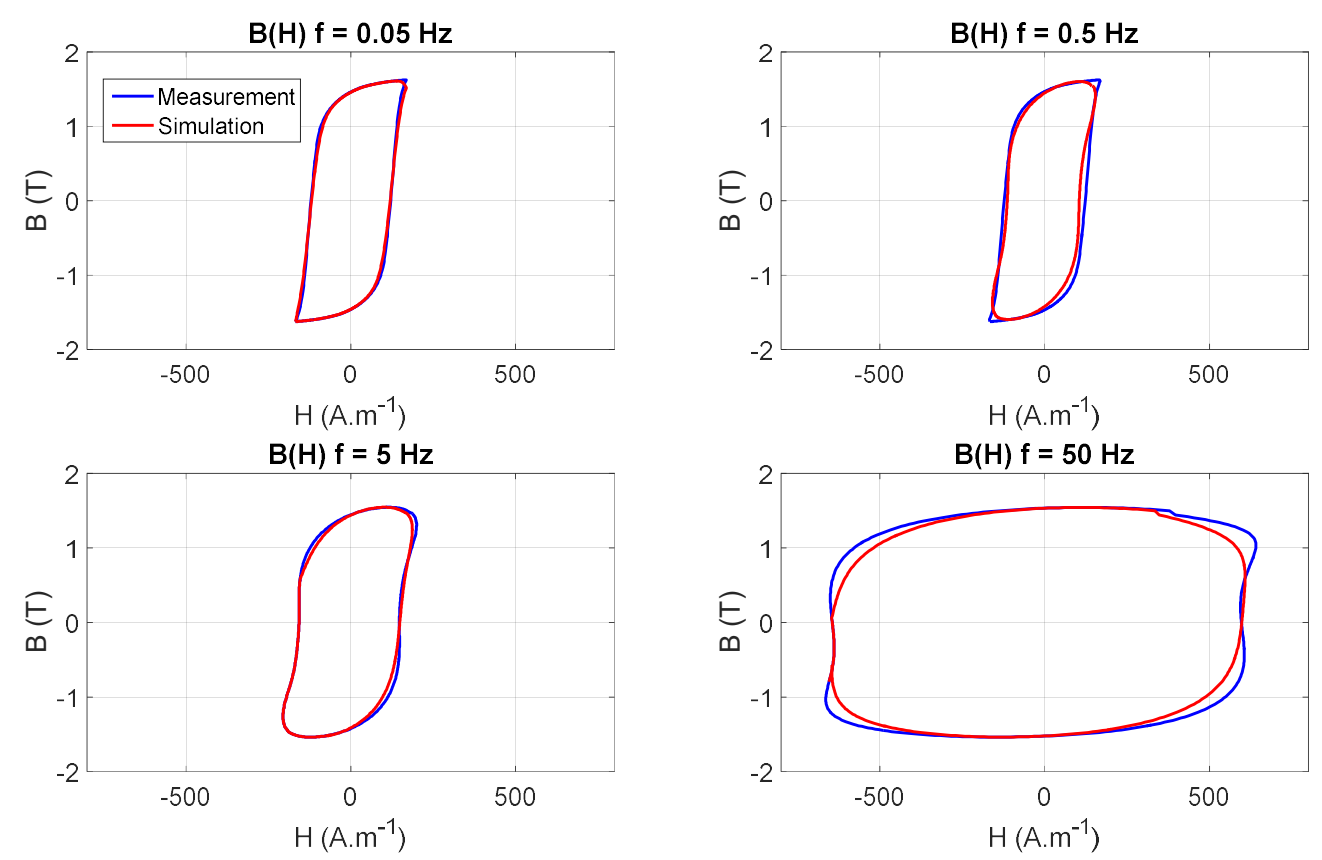

Fig. 10 - Comparative simulation/measurement for $\mathrm{B}(\mathrm{H})$ hysteresis loops $(f \varepsilon[0.05,50] \mathrm{Hz})$.

As in [8], we opted for the J-A model because of its simplicity and its limited number of parameters. The J-A model is described in detail in [34]-[36]. The J-A model parameter optimization process that we used is described in [37]. The static and dynamic simulation parameters are provided in Table 2.

Table 2 - Simulation parameters

\begin{tabular}{c|c} 
Static J-A Parameters & Typical value \\
\hline $\mathbf{a}$ & 41.56 \\
Ms (A.m $\left.{ }^{-1}\right)$ & $1.3810^{6}$ \\
k (A.m (1) $^{-1}$ & 290 \\
c & 0.19 \\
$\mathbf{\alpha}$ & $1.9410^{-4}$
\end{tabular}

\begin{tabular}{c|c} 
Dynamic parameters & Typical value \\
\hline $\boldsymbol{\sigma}\left(\mathrm{S}^{-1} \mathbf{m}^{-1}\right)$ & $4.210^{6}$ \\
$\boldsymbol{\alpha}$ & 0.64
\end{tabular}

Correct simulations results were observed over the entire tested frequency bandwidth. These comparisons provided a significant contribution to validate our theory. As predicted, the dynamic effects were massive. Unfortunately, experimental limitations appear early on the frequency scale and the highest experimental frequency that was reached was $50 \mathrm{~Hz}$.

Figure 11 shows the simulated current waveforms for all frequencies tested. We checked the unpredictable and distorted waveforms of the electrical currents as B was imposed in the tested magnetic ring. The experimental signals are not shown in Fig. 11; the current was forced and no difference could be observed. 


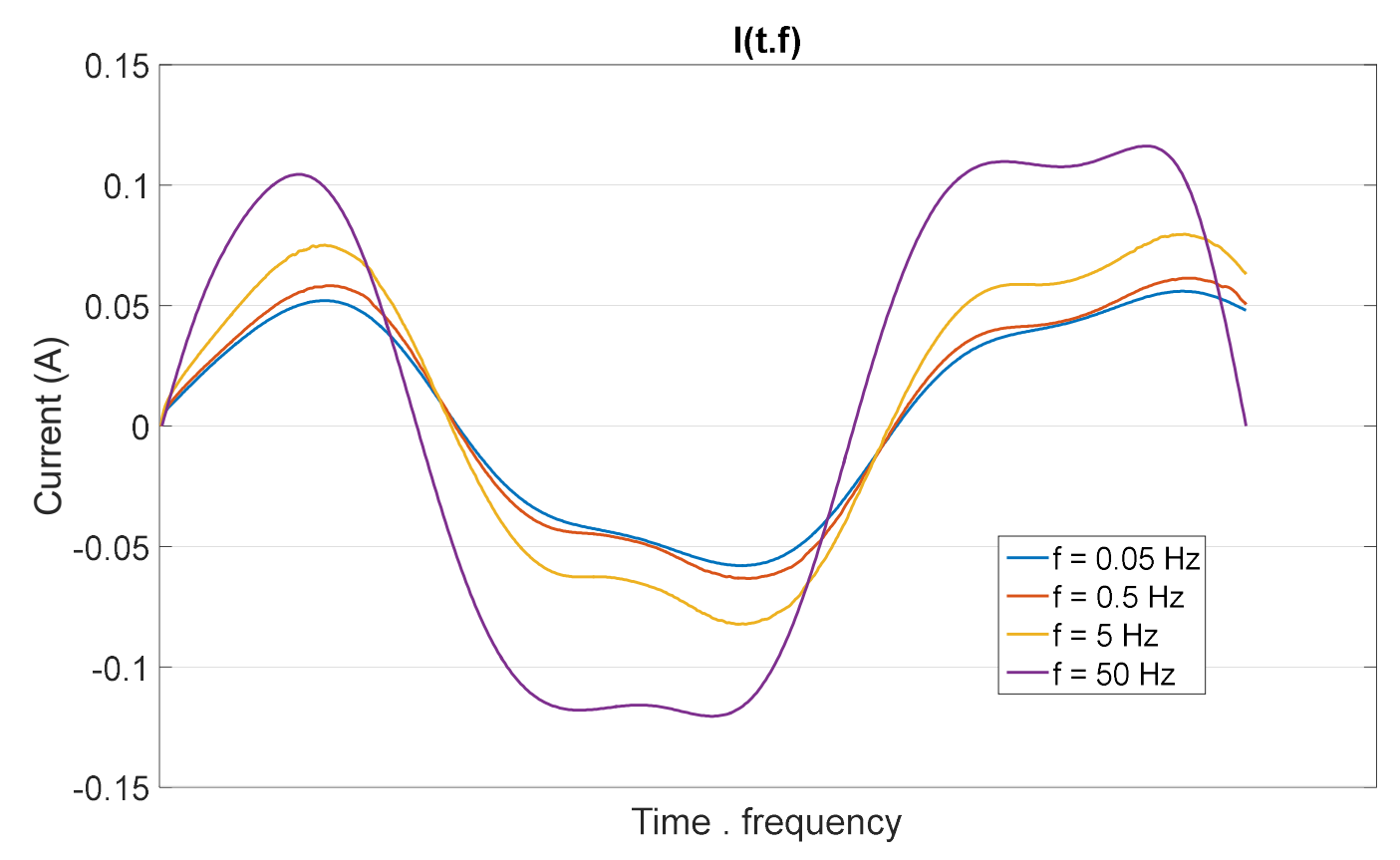

Fig. 11 - Current waveforms for different frequencies tested.

\section{5 - Conclusions}

Anomalous diffusion behaviors have been described in every branch of physics. The dynamic magnetic process of a toroidal massive magnetic core is one such diffusion behavior. The magnetic domain kinetics distort the magnetic diffusion field process, which becomes anomalous. This unfamiliar behavior requires the simulation of special mathematical operators. Such operators exist in the framework of fractional calculus. In this work, a 2D anomalous diffusion process through the cross section of a massive magnetic core was simulated with a modified magnetic field diffusion equation. The first-order time derivative term was replaced by a fractional term. Unlike the classic losses separation principle, the fractional diffusion equation lead to a simulation process that considered all dynamic contributions simultaneously and depended on a limited number of parameters:

_ $\sigma$ is the electrical conductivity that is imposed by the material nature that was obtained easily experimentally.

_ $\alpha$ is the fractional order that constitutes an additional degree of freedom of the simulation and that must be adjusted by comparative simulations/measurements. 
The modified fractional diffusion equation was solved through finite-difference space discretization combined with the Grünwald-Letnikov solution for the fractional derivative terms. For each simulation mesh node, a fractional equation must be solved. The numerical issues because of the implicit nature of the modified diffusion equation were limited because the Grünwald-Letnikov solution needs all simulated time steps of the function to be derived.

This simulation approach was particularly effective, and it provided local information as shown in Fig. 4. The simultaneous resolution agreed well and was coherent with the physical reality where the macroscopic and microscopic Eddy currents interacted continuously and could hardly be separated. In a previous article, this simulation approach was tested on a magnetic lamination. Here, we limited the simulation to a 2D geometrical situation. Future work should extend these geometry results where 3D simulations are mandatory. Electromagnetic non-destructive testing (NDT) that considers local defects (inclusions, cracks) in the simulation discretization could be an application for further exploration.

\section{Conflict of Interest: \\ Funding:}

This study was funded by the SCAC (Service de Cooperation et d'Action Culturelle) of the French Embassy to Cameroon.

- This study was funded by the National Natural Science Foundation of China (Grant No. 51805298) and by the Natural Science Foundation of Shandong Province (Grant No. ZR201807090390), Fundamental Research Funds for the Central Universities (Grant No. 2019ZRJC006). 


\section{References}

[1] J. Gyselinck, L. Vandevelde, P. Dular, C. Geuzaine, W. Legros, "A general method for the frequency demain FE modeling of rotating electromagnetic devices", IEEE Trans. on Mag., vol. 39, iss. 3, pp. 11471150, 2003.

[2] J. Letosa, J.S. Artal, M. Samplon, A. Uson, F.J. Arcega, "Modelization of current sensors by finite elements method", Measurement, vol. 35, iss. 3, pp. 233-241, 2004.

[3] B. Bao, Y. Zhou, P. Yang, G. Wang, "Theoritical and experimental study on novel weak current sensor using single nanocrystalline toroidal core with double-winding", vol. 141, iss. 1, pp. 84-88, 2008.

[4] J. Arcas, C. Gomez-Polo, M. Vazquez, A. Hernando, "Sensor applications based on induced magnetic anisotropy in toroidal cores", vol. 59, iss. 1-3, pp. 101-104, 1997.

[5] B. Orlando, R. Hida, R. Cuchet, M. Audoin, B. Viala, D. Pellissier-Tanon, X. Gagnard, P. Ancey, "Lowresistance integrated toroidal inductor for power management", vol. 42, iss. 10, pp. 3374-3376, 2006.

[6] H.J. Ryu, S.D. Kim, J.J. Lee, J. Kim, S.H. Han, H.J. Kim, C.H. Ahn, "2D and 3D simulation of toroidal type thin film inductors", IEEE Trans. on Mag., vol. 34, iss. 4, pp. 1360-1362, 1998.

[7] G. Bertotti, Hysteresis in Magnetism (Academic Press, Elsevier, New York, 1987).

[8] B. Ducharne, Y.A. Tene Deffo, B. Zhang, G. Sebald, "Anomalous fractional diffusion equation for magnetic losses in a ferromagnetic lamination", The Eur. Phys. J. Plus, 135:325, 2020.

[9] C.P. Steinmetz, “On the law of hysteresis", reprint. Proc. IEEE, vol. 72, iss. 2, pp. 196-221, 1984.

[10] G. Bertotti, "General properties of power losses in soft ferromagnetic materials", IEEE Trans. on Mag., vol. 24, iss. 1, pp. 621-630, 1980.

[11] M. A. Raulet, B. Ducharne, J.P. Masson, G. Bayada, "The magnetic field diffusion equation including dynamic hysteresis: a linear formulation of the problem", IEEE Trans. on Mag., vol. 40, iss. 2, pp. 872875, 2004.

[12] B. Ducharne, G. Sebald, D. Guyomar, G. Litak, "Fractional model of magnetic field penetration into a toroidal soft ferromagnetic sample", Int. J. of Dyn. and Cont., vol. 6, pp. 89-96, 2017.

[13] N. Engheia, "On the role of fractional calculus in electromagnetic theory", IEEE Antennas and Propagation Magazine, vol. 39, iss. 4, pp. 35-46, 1997.

[14] J.A. Tenreiro Machado, I.S. Jesus, A. Galhano, J. B. Cunha, "Fractional order electromagnetics", Signal Processing, vol. 86, iss. 10, pp. 2637-2644, 2006.

[15] R. Garrappa, "Grunwald-Letnikov operators for fractional relaxation in Havriliak-Negami models", Communication in Nonlinear Science and Numerical Simulation, vol. 38, pp. 178-191, 2016.

[16] D. Guyomar, B. Ducharne, G. Sebald, "The use of fractional derivation in modeling ferroelectric dynamic hysteresis behavior over large frequency bandwidth", Journal of Applied Physics, vol. 107, iss. 11, n 114108, 2010. 
[17] B. Zhang, B. Ducharne, G. Sebald, D. Guyomar, "Characterization of fractional order for highfrequency bandwidth model of dielectric ferroelectrics", Journal of Intelligent Material Systems and Structures JIMSS, vol. 27, iss. 4, pp. 437-443, 2016.

[18] B. Gupta, B. Ducharne, G. Sebald, T. Uchimoto, "A space discretized ferromagnetic model for nondestructive eddy current evaluation", IEEE Trans. on Mag., vol. 54, iss. 3, 2018.

[19] K.S. Miller, B. Ross, "An Introduction to the Fractional Calculus and Fractional Differential Equations", John Wiley \& Sons, New York, NY, USA, 1993.

[20] K.B. Oldham, J. Spanier, "The Fractional Calculus: Theory and Application of Differentiation and Integration to Arbitrary Order", Academic Press, New York, NY, USA, 1974.

[21] J.T. Machado, V. Kiryakova, F. Mainardi, "Recent history of fractional calculus", Communications in Nonlinear Science and Numerical Simulation, vol. 16, n³, pp. 1140-1153, 2011.

[22] B. Riemann, "Versuch Einer Allgemeinen Auffassung der Integration und Differentiation", Gesammelte Mathematische Werke und Wissenschaftlicher Nachlass, Teubner, Leipzig, 1876, Dover, New York, NY, USA, 1953.

[23] A.K. Grünwald, "Über "begrenzte" derivationen und deren anwendung", Zeitschrift für Mathematik und Physik, vol. 12, pp. 441-480, 1867.

[24] A.V. Letnikov, "Theory of differentiation with an arbitrary index", Sbornik: Mathematics, vol. 3, pp. 1-66, 1868 (Russian).

[25] M. Caputo, "Linear models of dissipation whose q is almost frequency independent-ii", Geophysical Journal of the Royal Astronomical Society, vol. 13, no. 5, pp. 529-539, 1967.

[26] B. Zhang, B. Gupta, B. Ducharne, G. Sebald, T. Uchimoto, "Dynamic magnetic scalar hysteresis lump model, based on Jiles-Atherton quasi-static hysteresis model extended with dynamic fractional derivative contribution", IEEE Trans. on Mag., vol. 54, iss. 11, pp. 1-5, 2018.

[27] B. Zhang, B. Gupta, B. Ducharne, G. Sebald, T. Uchimoto, "Preisach's model extended with dynamic fractional derivation contribution", IEEE Trans. on Mag., vol. 54, iss. 3, 2017.

[28] B. Ducharne, B. Newell, G. Sebald, "A unique fractional derivative operator to simulate all dynamic piezo ceramic dielectric manifestations: from aging to frequency dependent hysteresis", IEEE Transactions on Ultrasonic, Ferroelectricity and Frequency Control, vol. 67, iss. 1, pp. 197-206, 2019.

[29] IEC 60404-6, Magnetic materials - Part 6: Methods of measurement of the magnetic properties of magnetically soft metallic and powder materials at frequencies in the range $20 \mathrm{~Hz}$ to $100 \mathrm{kHz}$ by the use of ring specimens. Int. Elect. Com., 2018.

[30] B. Ducharne, G. Sebald, D. Guyomar, G. Litak, "Dynamics of magnetic field penetration into soft ferromagnets", J. Appl. Phys., vol. 117, iss. 24, 243907, 2015.

[31] J.V. Leite, N. Sadowski, P. Kuo-Peng, N.J. Batistela, J.P.A. Bastos, "The inverse Jiles-Atherton model parameters identification", IEEE Trans. on Mag., vol. 39, iss. 3, pp. 1397-1400, 2003.

[32] J.V. Leite, N. Sadowski, P. Kuo-Peng, N.J. Batistela, J.P.A. Bastos, A.A. de Espindola, "Inverse JilesAtherton vector hysteresis model", IEEE Trans. on Mag., vol. 40, iss. 4, pp. 1769-1775, 2004. 
[33] E. Cardelli, E.D. Torre, B. Tellini, "Direct and inverse Preisach modeling of soft materials", IEEE Trans. on Mag., vol. 36, iss. 4, pp. 1267-1271, 2000.

[34] D.C Jiles, D.L Atherton, "Theory of ferromagnetic hysteresis", J. of Mag. and Mag. Mat., vol. 61, pp. 48-60, 1986.

[35] J.V. Leite, S.L. Avila, N.J. Batistela, W.P. Carpes, N. Sadowski, P. Kuo-Peng, J.P.A Bastos, "Real coded genetic algorithm for Jiles-Atherton model parameters identification", vol. 40, iss. 2, pp. 888-891, 2004.

[36] D. Jiles, "Introduction to magnetism and magnetic materials", Chapman \& Hall, New York, 1991.

[37] B. Gupta, B. Ducharne, T. Uchimoto, G. Sebald, T. Miyazaki, T. Takagi, “Non-destructive testing on creep degraded 12\% Cr-Mo-W-V ferritic test samples using Barkhausen noise", J. of Mag. and Mag. Mat., vol. 498, n 166102, 2020. 